\section{Case Reports in Nephrology and Dialysis}

\title{
Statin-Associated Necrotizing Myopathy Leading to Acute Kidney Injury: A Case Report
}

\author{
Tadej Petreski ${ }^{a, b} \quad$ Nejc Piko ${ }^{a, c}$ Timotej Petrijan ${ }^{d}$ Benjamin Dvoršak ${ }^{a, b}$ \\ Radovan Hojs ${ }^{a, b}$ Sebastjan Bevc ${ }^{a, b}$
}

aDepartment of Nephrology, Clinic for Internal Medicine, University Medical Center Maribor, Maribor, Slovenia; ${ }^{b}$ Medical Faculty, University of Maribor, Maribor, Slovenia; 'Department of Dialysis, Clinic for Internal Medicine, University Medical Center Maribor, Maribor, Slovenia; dDepartment of Neurology, University Medical Center Maribor, Maribor, Slovenia

\section{Keywords}

Statin · Acute kidney injury · Necrotizing myopathy · High cutoff hemodialysis ·

Rhabdomyolysis

\begin{abstract}
Statins or 3-hydroxy-3-methylglutaryl coenzyme A reductase inhibitors are a mainstay of cardiovascular disease therapy. In addition to their lipid-lowering capabilities, they exhibit several pleiotropic effects. Their adverse reactions such as myalgias are not uncommon, but in rare cases, the resulting rhabdomyolysis can be fatal. Recently, more insight has been brought into the pathogenesis of statin-induced rhabdomyolysis, and immune-mediated necrotizing myopathies are diagnosed more frequently. We present a case of a female patient who was on chronic rosuvastatin therapy and developed necrotizing myopathy. The disease progressed to acute kidney and liver injury. We discontinued the drug, started supportive measures, and initiated renal replacement therapy with a high cutoff dialysis membrane once. Her recovery was prompt, with a normal control electromyography 2 weeks after discharge.
\end{abstract}

(C) 2021 The Author(s).

Published by S. Karger AG, Basel

\section{Introduction}

Rhabdomyolysis is a condition of muscle cell injury resulting from different causes. Regardless of the etiologic factor, its conventional presenting triad, which is present in $<10 \%$ cases, consists of myalgia, muscle weakness, and dark colored urine $[1,2]$. 
Current recommendations suggest using creatine kinase (CK) $>5 \times$ upper limit of normal as the definition of mild rhabdomyolysis. Severe rhabdomyolysis is combined with myoglobinuria and/or acute kidney injury (AKI) [1]. Serum measurement of myoglobin is not always required for diagnosis as it has a shorter half-life compared to CK (3 vs. $36 \mathrm{~h}$ ) [2].

AKI develops in $10-40 \%$ of patients with severe rhabdomyolysis [3]. The main pathogenetic mechanism is precipitation of Tamm-Horsfall protein in kidney tubules, which interacts with myoglobin and forms granular casts that cause tubular obstruction and consequent renal injury [2,3]. Additionally, muscle injury can cause extravasation of fluid into the interstitial space and produce free radicals, both important in understanding the pathophysiology of AKI as well $[3,4]$. Here, we present a case of statin-associated necrotizing myopathy, which leads to AKI and acute liver injury, that was treated with statin discontinuation, supportive measures, and a single-time hemodialysis with a high cutoff (HCO) dialysis membrane.

\section{Case Report}

A 59-year-old Caucasian female was transferred to the Department of Nephrology, University Medical Centre Maribor, due to rhabdomyolysis-induced acute kidney and liver injury. She began treatment in another hospital, where they already prescribed infusions of balanced crystalloids, ceftriaxone intravenously for a urinary infection with $E$. coli, sodium bicarbonate for metabolic acidosis, folic acid and vitamin D supplementation, and calcium carbonate tablets due to hyperphosphatemia.

Her past medical history revealed arterial hypertension and dyslipidemia, both for about 5 years, which were treated with perindopril/indapamide 4/1.25 mg and rosuvastatin $40 \mathrm{mg}$. Additionally, she was taking over-the-counter preparations of milk thistle (Silybum marianum) $120 \mathrm{mg}$ daily for the last month. She had no prior history of smoking or alcohol consumption.

At admission, she reported muscle aches in her calves that gradually spread over all her body, and she had difficulty walking up and down the stairs and standing up from a sitting position. As her symptoms progressed, she noticed darkening of her urine. She denied exertional dyspnea or orthopnea, any recent trauma, strenuous physical activity, having fever, rigors, or painful micturition.

Physical examination showed an euvolemic patient, with a silent systolic murmur (grade 1/6) over Erb's point and presence of mild pretibial edema. Neurologic examination revealed proximal (grade 3-4/5 on MRC scale) and distal (grade 4/5 on MRC scale) muscle weakness with prominent weakness of torso flexors.

Initial laboratory tests revealed several pathologic findings, which are presented in Table 1 . The urine sample showed proteinuria $(1+)$, microscopic examination of sediment 6-10 leucocytes, and 6-10 erythrocytes per high-power field, and no casts were seen. Her TSH was within reference range. Additionally, several immunologic tests (ANA, ANCA, AMA, anti-ENA, anti-GBM, anti-SS-A, anti-SS-B, anti-SM, and anti-Scl-70), a liver profile (M2/n, M2/OGDC-E2, M2/BCOADC-E2, M2/PDC-E2, gp210, sp100, LKM1, LC1, SLA, and F-actin), and a myositis profile (Jo-1, PL-7, PL-12, EJ, SRP, Mi-2, MDA-5, Tif1-gamma, HMGCR, Ro-52, SAE1/SAE2, and NXP-2) were done, which were all negative (abbreviations explained in Appendix 1).

The chest X-ray and ultrasound scan of the abdomen showed no pathologic findings. The electromyography revealed electrophysiologic signs of a generalized, inflammatory, or necrotizing myopathy with signs of abundant pathological spontaneous activity in the form of fibrillation potentials and positive sharp waves and predominantly small, short-lasting, and polyphasic muscle unit action potentials in proximal and distal muscles.

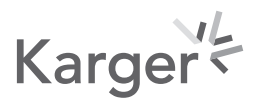




\section{Case Reports in Nephrology and Dialysis}

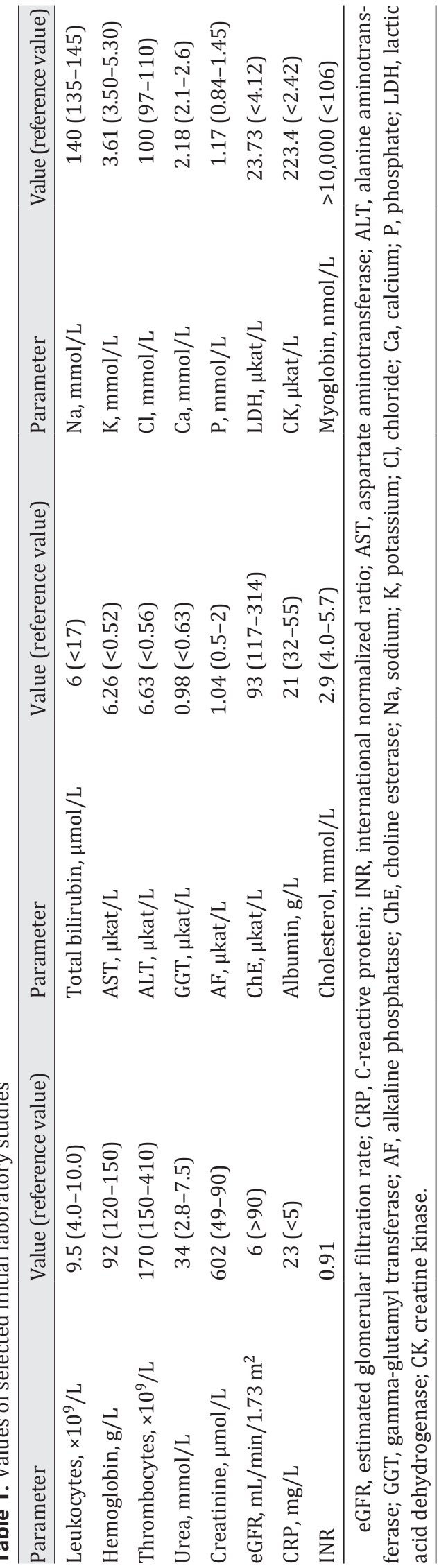

\section{Karger'}


Table 2. Serial values of kidney and liver function tests, muscle enzymes, and albumin

\begin{tabular}{lllllllll}
\hline & Day 1 & $\begin{array}{l}\text { Day 1 (after HD } \\
\text { with HCO) }\end{array}$ & Day 2 & Day 4 & Day 6 & Day 9 & Day 11 & Day 13 \\
\hline Urea, mmol/L & 34 & 5.2 & 7.9 & 9.8 & 8.2 & 5.4 & 5.6 & 4.8 \\
Creatinine, $\mu$ mol/L & 602 & 134 & 227 & 332 & 311 & 240 & 204 & 182 \\
AST, $\mu$ kat/L & 6.26 & & & 1.41 & 0.79 & & 0.33 & \\
ALT, $\mu$ kat/L & 6.63 & & & 3.04 & 2.12 & & 0.33 & \\
GGT, $\mu$ kat/L & 0.98 & & & 0.75 & 2.12 & & 0.87 & \\
AF, $\mu$ kat/L & 1.04 & & & 0.93 & 1.12 & & 1.25 & \\
CK, $\mu$ kat/L & 223.4 & & 103.69 & 29.77 & 9.09 & 2.95 & 1.88 & 1.28 \\
Myoglobin, nmol/L & $>10,000$ & 4,998 & 9,797 & 1,961 & 574 & 230 & 164 & 139 \\
Albumin, g/L & 21 & 23 & & 30 & 29 & & 31 & \\
\hline
\end{tabular}

AST, aspartate aminotransferase; ALT, alanine aminotransferase; GGT, gamma-glutamyl transferase; AF, alkaline phosphatase; $\mathrm{CK}$, creatine kinase; HD with $\mathrm{HCO}$, hemodialysis procedure with a high cutoff membrane dialyzer.

After admission, she continued with previous treatment; additionally, she received humane albumin infusions for hypoalbuminemia. We performed a hemodialysis procedure with a HCO membrane dialyzer (Theralite; Baxter) on day 1, and afterwards no further hemodialysis procedure was needed. Additionally, we performed a biopsy of the deltoid muscle, which showed a necrotizing myopathy, with evenly distributed signs of muscle fiber injury and presence of macrophages, while no lymphocytes were found. The pathologist concluded that it could be a statin-induced immune necrotizing myopathy due to mildly enhanced MHC class I and C5b-9 complement complex expression.

During our treatment, the patient slowly became polyuric on day 3 , with daily diuresis of about $4.5 \mathrm{~L}$, which lasted for several days. We observed a fall in serum creatinine, myoglobin, $\mathrm{CK}$, and liver enzymes. As the patient was already recovering when we received the biopsy results, no immunosuppressive therapy was initiated. Upon discharge, she reported less muscle aches and was feeling well in general. Serial laboratory measurements are presented in Table 2.

After 3 weeks, a repeat electromyography was performed that showed individual myopathic muscle unit action potentials in proximal muscles without signs of active denervation. The neurologic exam revealed no muscle atrophy or muscle weakness.

\section{Discussion}

Since their discovery in 1976, statins or 3-hydroxy-3-methylglutaryl coenzyme A (HMG$\mathrm{CoA}$ ) reductase inhibitors have revolutionized the therapy of cardiovascular disease [5]. In 2012 , nearly one-quarter (23.2\%) of US adults over 40 years reported use of statins [6]. They are mainly used for their lipid-lowering effect, targeting primarily low-density lipoprotein; however, they provide additional cardiovascular protection with pleiotropic effects such as stabilization of the atheroma plaque, improvement of endothelial function, anti-inflammatory and immunomodulatory effects, and reducing platelet activity [5].

On the other hand, every medication has its adverse effects. Statin use has mostly been associated with myopathy and rhabdomyolysis, development of diabetes mellitus, and hemorrhagic stroke, while some studies suggest additional effects, but are not very compelling [5]. Overall adverse event rate is $<0.5 \%$, with muscle injury presenting $<0.1 \%$; however, this could be under- 
reported [7]. Patients are at highest risk the first 120 days after initiation of statin treatment. A proposed mechanism for rosuvastatin-induced rhabdomyolysis is CYP2C9 enzyme saturation [4].

A review on management of rhabdomyolysis in the emergency department has listed several etiologies for rhabdomyolysis, such as trauma, exertion, changes in body temperature, electrolyte abnormalities, muscle ischemia, infections, drugs, endocrine, autoimmune, and genetic causes [2]. In our case, we believe the main culprit to be rosuvastatin which caused a necrotizing myopathy, as we have excluded most causes except genetic, which we think are unlikely, especially with the patients age of 59 years and negative family history.

As rosuvastatin seemed the most likely causative agent, we have checked several resources for drug interactions between rosuvastatin, perindopril, and indapamide. Our search yielded no known interactions, and additionally some pharmaceutic companies develop polypills with this drug combination. Furthermore, we have explored the literature for effects of milk thistle (S. marianum). A recent review reported only few adverse reactions, such as gastric upset, when taking silymarin, a major constituent of the milk thistle extract [8]. Moreover, it does not appear to affect rosuvastatin pharmacokinetics in vivo [9].

Statin-associated myopathies are clinically divided into 4 groups: rhabdomyolysis, myalgia and/or mildly elevated CK, self-limited toxic myopathy, and immune-mediated necrotizing myopathy [10]. Recently, data are emerging on immune-mediated necrotizing myopathies, which are composed of antisignal recognition particle, anti-HMG-CoA reductase, and autoantibody-negative immune-mediated necrotizing myopathy [11]. Statins are associated with anti-HMG-CoA reductase myopathy, where the incidence is estimated to be 2 cases per million per year. A recent case series identified the average age of patients to be around 65 years, and most of them were on chronic statin therapy for several years [6]. They usually present with proximal muscle weakness that does not improve with statin cessation. The treatment usually consists of immunosuppressive therapy, where most patients need 2 or more immunosuppressants. A working group has recommended corticosteroids and methotrexate as an initial regimen $[6,11]$. In our patient, the muscle biopsy has shown signs of necrotizing myopathy with presence of macrophages, MHC class I, and C5b-9 complement complex, which are usually present in immune-mediated necrotizing myopathies; however, all antibodies we tested proved to be negative. According to the biopsy, this could still be a case of autoantibody-negative immune-mediated necrotizing myopathy. Nevertheless, as our patient was improving very well after statin discontinuation and was almost asymptomatic when we received the biopsy results, we have decided against immunosuppressive therapy. With all available information, spontaneous improvement after statin cessation, AKI, and absent autoantibodies, we believe this was a case of severe self-limited statin myopathy (statin-induced rhabdomyolysis), which affects $<1$ in 100,000 treated patients. Statins should not be administered again for this patient in the future, if need be proprotein convertase subtilisin/kexin type 9 (PCSK9) inhibitors can be used as an alternative [10].

The mainstay therapy of rhabdomyolysis is expansion of intravascular volume and removal of the underlying cause. Early and aggressive volume resuscitation should be initiated targeting urine output of $200-300 \mathrm{~mL} / \mathrm{h}$. Currently, there is no evidence that using balanced crystalloids is better than normal saline; however, the rationale is that saline can cause metabolic acidosis which lowers myoglobin clearance. For the same reason, urinary alkalinization with sodium bicarbonate has been proposed, although it is not evidence based. Additionally, forced diuresis with mannitol or furosemide is generally not recommended [2,3]. We have stopped rosuvastatin at admission and continued with infusions of balanced crystalloids and sodium bicarbonate, which was principally given for metabolic acidosis prior to admission at our hospital but could have provided additional benefit for myoglobin clearance. It was discontinued afterwards as her pH levels of capillary blood rose to slight alkalosis. Additionally, ACE inhibitors, such as perindopril in this case, are also often associated with AKI, and guidelines

\section{Karger's}


suggest stopping them in AKI, which we did. However, Thomson and Tomlinson [12] wrote an article discussing whether stopping renin-angiotensin system inhibitors is always necessary or not, as trials have shown conflicting data.

If patients remain anuric or develop other life-threatening complications of AKI, thought should be given to start renal replacement therapy (RRT). Typically, 4-20\% of patients with AKI due to rhabdomyolysis require RRT, where continuous methods are preferred [2,3]. As standard hemodialysis procedures have limited myoglobin removal, a novel approach with the use of HCO RRT has been suggested in recent years, although no strong evidence for improving patient outcomes exists yet. HCO RRT has up to 20 -fold higher clearances of myoglobin compared to standard RRT. Although due to larger pore size, one must account for loss of some other molecules such as cytokines, coagulation factors, and albumin as well [13]. Because of fast recovery of kidney function, prerenal azotemia might have been the predominant factor in the patient's AKI, as AKI due to rhabdomyolysis is usually caused by acute tubular necrosis, which takes longer to resolve. This is one of the reasons why the use of HCO RRT was especially beneficial for our patient because we have removed myoglobin before the kidneys were injured by it and prevented acute tubular necrosis. As we have already started with albumin infusions due to severe hypoalbuminemia, further loss with use of HCO RRT was anticipated, which is evident from the serum albumin levels after RRT. Additionally, we have used HCO RRT only once, which limited its known risks significantly.

\section{Conclusion}

Due to a global rise of cardiovascular disease, statins will remain broadly prescribed for their several positive effects that outweigh their costs. However, practitioners need to be vigilant for their adverse reactions. If a patient presents with more than just transient myalgia, especially with muscle weakness, rhabdomyolysis should be considered. In case of uncommon clinical manifestations, specific diagnostic and treatment are required.

\section{Acknowledgement}

We would like to acknowledge all our coworkers at the Department of Nephrology and the Department of Dialysis, University Medical Center Maribor, Slovenia, for their constant support and help.

\section{Statement of Ethics}

Written consent for publication of the case was obtained from the patient.

\section{Conflict of Interest Statement}

The authors have no conflicts of interest to declare.

\section{Funding Sources}

The authors have no funding sources to declare.

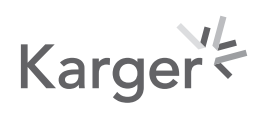




\section{Case Reports in Nephrology and Dialysis}

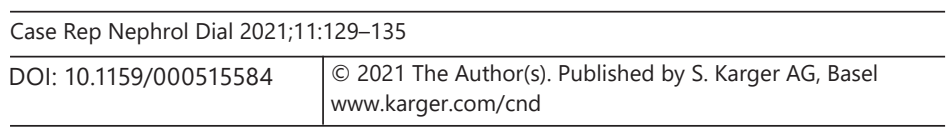

Petreski et al.: A Case of Statin-Induced Rhabdomyolysis

\section{Author Contributions}

All authors have been involved in patient treatment and have equally contributed to the writing of the manuscript.

\section{Appendix 1}

\section{List of Immunologic Test Abbreviations}

ANA, anti nuclear antibody; ANCA, anti neutrophil cytoplasmic antibody; AMA, anti mitochondrial antibody; Anti-ENA, anti extractable nuclear antigen; Anti-GBM, anti glomerular basement membrane; Anti-SS-A, anti Sjogren syndrome related antigen A; Anti-SS-B, anti Sjogren syndrome related antigen B; Anti-SM, anti Sm antigen; Anti-Scl-70, anti topoisomerase-I; M2 /n, anti E1, E2, E3 subunits of pyruvate dehydrogenase complex; M2/OGDC-E2, anti E2 subunit of oxoglutarate dehydrogenase complex; M2/BCOADC-E2, anti E2 subunit of branched-chain oxo acid dehydrogenase complex; M2/PDC-E2, anti E2 subunit of pyruvate dehydrogenase complex; Gp210, anti glycoprotein 210; Sp100, anti Sp100 nuclear antigen; LKM1, anti liver kidney microsomal type 1; LC1, anti liver cytosol 1; SLA, anti soluble liver antigen; Jo-1, anti jo-1 antigen; PL-7, ani threonyl tRNA synthetase; PL-12, anti alanyl tRNA synthetase; EJ, anti glycyl tRNA synthetase; SRP, anti signal recognition particle; Mi-2, anti mi-2 nuclear antigen; MDA-5, anti melanoma differentiation-associated gene 5; Tif1-gamma, anti transcription intermediary factor 1 gamma; HMGCR, anti 3-hydroxy-3-methylglutaryl coenzyme A reductase; Ro-52, anti Ro-52 protein; SAE1/SAE2, anti SUM01 activating enzyme subunit 1 and 2; NXP-2, anti nuclear matrix protein 2.

\section{References}

1 Stahl K, Rastelli E, Schoser B. A systematic review on the definition of rhabdomyolysis. J Neurol. 2020;267(4): 877-82.

2 Long B, Koyfman A, Gottlieb M. An evidence-based narrative review of the emergency department evaluation and management of rhabdomyolysis. Am J Emerg Med. 2019;37(3):518-23.

3 Esposito P, Estienne L, Serpieri N, Ronchi D, Comi GP, Moggio M, et al. Rhabdomyolysis-associated acute kidney injury. Am J Kidney Dis. 2018;71(6):A12-a4.

4 Suthar KS, Vanikar AV, Trivedi HL. Acute kidney injury and quadriparesis due to rosuvastatin induced rhabdomyolysis: a case report. J Clin Diagn Res. 2015;9(5):Od08-9.

5 Pinal-Fernandez I, Casal-Dominguez M, Mammen AL. Statins: pros and cons. Med Clin. 2018;150(10):398402.

6 Nazir S, Lohani S, Tachamo N, Poudel D, Donato A. Statin-associated autoimmune myopathy: a systematic review of 100 cases. J Clin Rheumatol. 2017;23(3):149-54.

7 Mohamed MFH, Salameh OK, Saeed AAM. Statin-induced rhabdomyolysis, acute kidney injury, and hepatitis leading to death. Am J Case Rep. 2019;20:709-12.

8 Soleimani V, Delghandi PS, Moallem SA, Karimi G. Safety and toxicity of silymarin, the major constituent of milk thistle extract: an updated review. Phytother Res. 2019;33(6):1627-38.

9 Deng JW, Shon JH, Shin HJ, Park SJ, Yeo CW, Zhou HH, et al. Effect of silymarin supplement on the pharmacokinetics of rosuvastatin. Pharm Res. 2008;25(8):1807-14.

10 Selva-O'Callaghan A, Alvarado-Cardenas M, Pinal-Fernández I, Trallero-Araguás E, Milisenda JC, Martínez M, et al. Statin-induced myalgia and myositis: an update on pathogenesis and clinical recommendations. Expert Rev Clin Immunol. 2018;14(3):215-24.

11 Pinal-Fernandez I, Casal-Dominguez M, Mammen AL. Immune-mediated necrotizing myopathy. Curr Rheumatol Rep. 2018;20(4):21.

12 Tomson C, Tomlinson LA. Stopping RAS inhibitors to minimize AKI: more harm than good? Clin J Am Soc Nephrol. 2019;14(4):617-9.

13 Heyne N, Guthoff M, Krieger J, Haap M, Häring HU. High cut-off renal replacement therapy for removal of myoglobin in severe rhabdomyolysis and acute kidney injury: a case series. Nephron Clin Pract. 2012;121(34):c159-64. 